\title{
Crater on Ganymede emplaced at the border of the dark and bright terrain - view into the subsurface properties
}

\author{
Katrin Krohn ${ }^{1}$, Katrin Stephan ${ }^{1}$, Roland Wagner ${ }^{1}$, Namitha Rose Baby ${ }^{1}$, and Nico Schmedemann ${ }^{2}$ \\ ${ }^{1}$ German Aerospace Center (DLR), Institute of Planetary Research, Berlin, Germany (katrin.krohn@dlr.de) \\ ${ }^{2}$ Institut für Planetologie, Westfälische Wilhelms-Universität Münster, Germany
}

Two major terrain units dominate Ganymede's surface: 1) the ancient heavily cratered dark terrain and 2) the tectonically resurfaced bright or light terrain (Collins et al., 2013). Previous studies suggest that the dark material is a relatively thing lag deposition overlying a brighter ice-rich subsurface formed by processes such as impact gardening, thermal segregation, and mass wasting (Prockter et al., 1998). The younger bright or light terrain cross cutting the older dark terrain formation is believed to have formed predominantly through the modification of dark terrain by tectonic and possibly also cryovolcanic resurfacing processes (Collins et al., 1998). Particularity, the better understanding of the formation of the bright terrain and its possible interaction with a subsurface ocean makes its investigation one of the top goals of the upcoming JUICE mission (Grasset et al., 2013). In order to prepare for the JUICE mission and maximizing its science return, we started the re-investigated the available Galileo SSI and NIMS data set. In this work, we focus on Ganymede's numerous impact craters that have been directly emplaced at the border of both terrains. Often fresh impact craters and their ejected material offer a glimpse into the subsurface properties. Therefore, the study of the selected impact craters could enable to reveal any changes in the bright terrain properties during its formation compared to the subsurface of the dark terrain. Variations in the subsurface properties could be indirectly mirrored in the morphology and surface texture of the crater material, the superimposed crater density but also the composition of the crater material excavated for the subsurface.

We present a detailed analysis of these craters based on Galileo SSI images focusing on morphology, albedo, surface texture of the crater material accompanied by surface age measurements.

These investigated impact craters are distributed across Ganymede's entire surface and include ancient palimpsests and penepalimpsest, impact craters of different morphologies including pit, dome and pedestal craters, but also fresh dark ray and bright crater craters as well crater chains (catenea) with diameters from a few to about hundreds of kilometers. All investigated impact craters superimpose both dark and bright terrain and thus have been emplaced during or after the bright terrain formation.

One of the most prominent and best imaged examples of these kind of impact craters is Melkart $\left(\sim 10^{\circ} \mathrm{S} / 174^{\circ} \mathrm{E}\right)$, which is located directly at the border between the ancient dark terrain of Marius Regio and a N-S trending branch of younger bright terrain extending from Sippar Sulcus (Fig.1). The central dome is affected by fractures and lineaments, which are thought to be caused by stretching 
of the outer skin (Schenk 2010). Furthermore, the dome is surrounded by an irregular depression of smooth material. The boundary between the bright and the dark material is displayed by a tectonic lineament crosses the crater directly along its central dome and straightened the dome slightly in NNW- ESE direction. The lineament not only crosses the dome, where it is more prominent, but also the knobby crater floor (Fig.2).

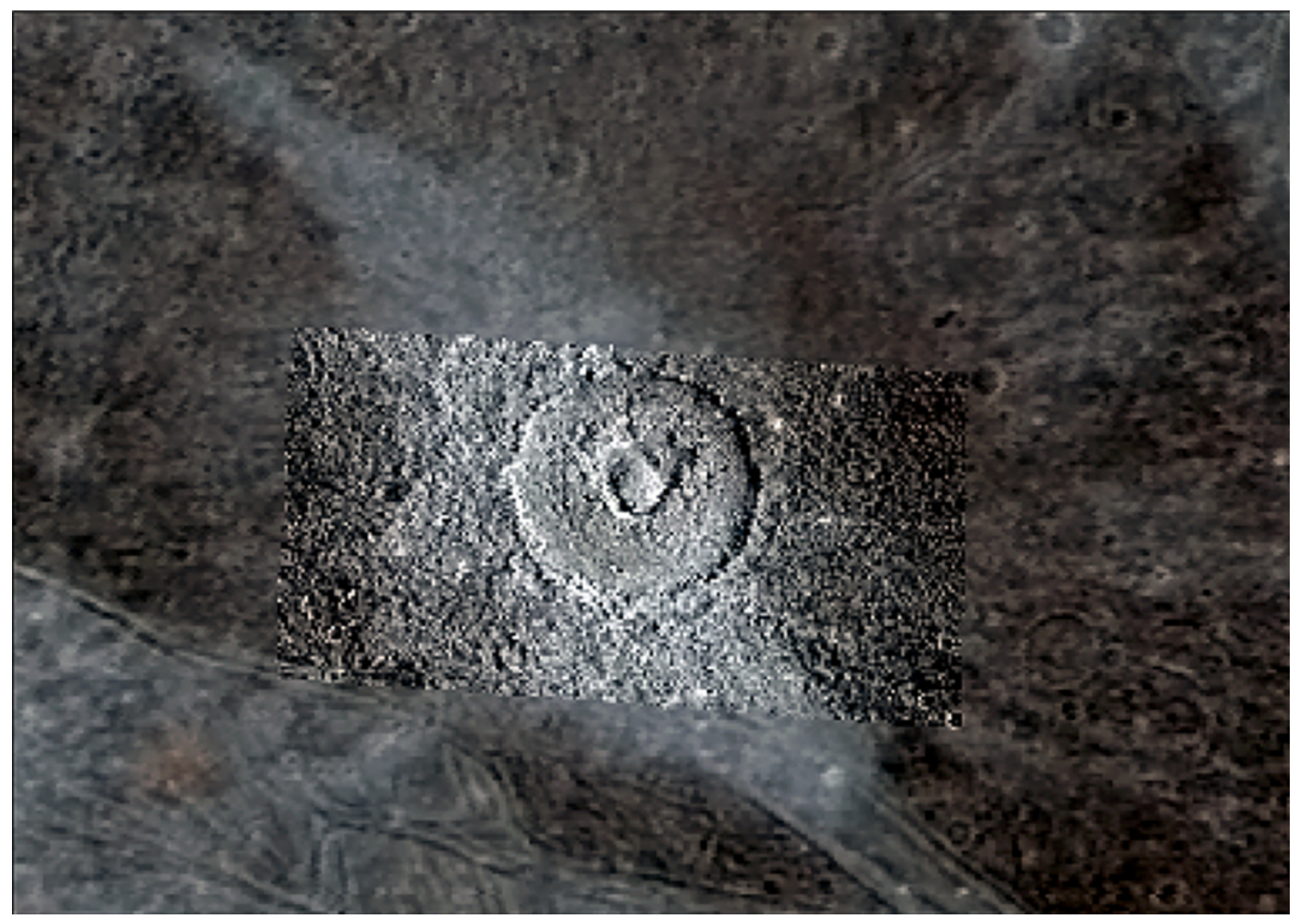

Fig. 1: Voyager-Galileo SSI color mosaic of Melkart crater showing the dark bright boundary. 


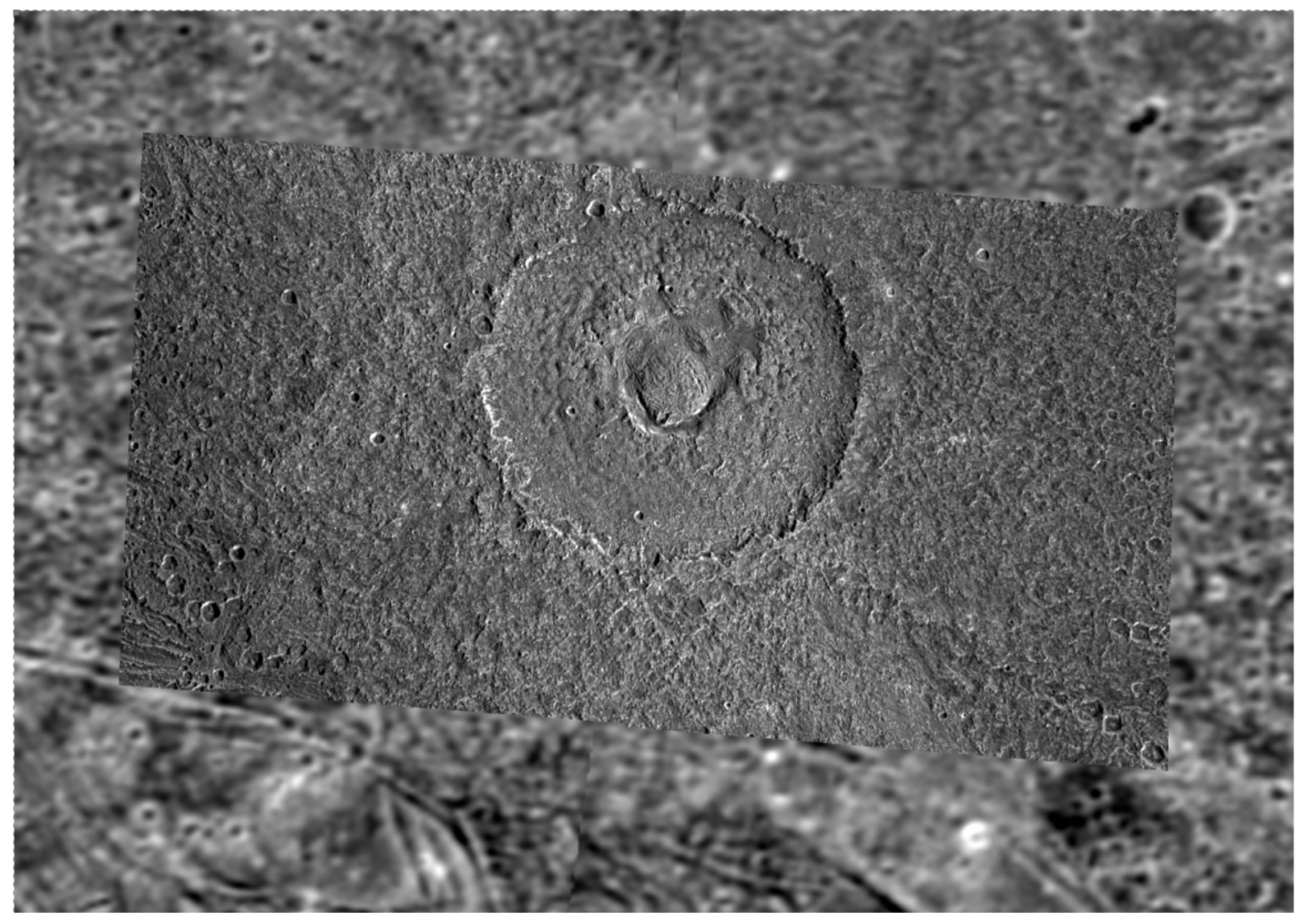

Fig. 2: Type locality of impact craters emplaced at the border of bright and dark terrain: Dome crater Melkart imaged by Galileo SSI (G8GSMELKRT01).

\section{References:}

Collins, G. C. et al. (1998), Formation of Ganymede Grooved Terrain by Sequential Extensional Episodes: Implications of Galileo Observations for Regional Stratigraphy: Icarus, 135, 1, 345-359.

Collins, G. C. et al. (2013) Global geologic map of Ganymede, p. 3237.

Grasset, O. et al. (2013) JUpiter ICy moons Explorer (JUICE): An ESA mission to orbit Ganymede and to characterise the Jupiter system: PSS, 78, 1-21.

Stephan, K. et al. (2008) Ganymede's Impact Crater Melkart: An Example for a Combination of HighResolution Spectral and Geological Analyses in the Outer Solar System, The Science of Solar System Ices (ScSSI): A Cross-Disciplinary Workshop: Houston, Lunar and Planetary Institute, Abstract \#9060.

Prockter, L. M. et al. (1998) Dark Terrain on Ganymede: Geological Mapping and Interpretation of Galileo Regio at High Resolution: Icarus, 135, 1, 317-344.

Schenk, P. (2010) Atlas of the Galilean Satellites 